\title{
SU(2) coherent states in parametric down-conversion
}

\author{
A. Luis ${ }^{*}$ and J. Peřina \\ Laboratory of Quantum Optics, Palacký University, 17. listopadu 50, 77207 Olomouc, Czech Republic
}

(Received 7 September 1995)

\begin{abstract}
The states generated from the vacuum in two parametric down-conversion crystals with aligned idler beams are studied. It is shown that after a measurement of the photon number in some of the modes, the emerging states can be related to $\mathrm{SU}(2)$ and $\mathrm{SU}(1,1)$ coherent and minimum-uncertainty states.

PACS number(s): 42.50.Dv
\end{abstract}

\section{INTRODUCTION}

The entangled nature of the two-mode field generated by parametric down-conversion has been widely studied and utilized in a number of fundamental experiments in quantum optics [1].

On the other hand, entanglement is a powerful tool concerning both quantum measurement and the generation of particular system states. In this sense measurement is not only the way to obtain information but also a way to manipulate a system state by means of a proper choice of the measurement and the corresponding selection of its outputs [2].

In the context of parametric down-conversion it has been shown how a measurement in the idler mode can be useful to transfer desirable properties to the state in the signal mode [3-5]. Specifically, the measurement of the photon number in the idler mode leads to near-number states in the signal mode.

Recently parametric down-conversion has been used to show an interference experiment exhibiting nonclassical features [6]. Two nonlinear crystals were pumped coherently to produce two pairs of signal and idler beams via spontaneous parametric down-conversion. The signal beams were mixed at a beam splitter looking for interference. When the two down-converters are unconnected, the signal field shows no mutual second-order coherence in complex amplitudes. However, interference is observed when the two idler beams are aligned, the idler beam of one of the crystals passing through the second one. The interference is lost whenever this connection is blocked.

This behavior has been explained on the basis of the relation between coherence and quantum indistinguishability $[6,7]$. When the idler beams are unconnected, there exists the possibility, in principle, of identifying the path of each signal photon reaching the beam splitter, knowledge that wipes out the interference. When the idlers are connected this possibility can be ruled out, giving rise to interference. The entanglement, which provides the which-path information is then reverted into coherence. After the alignment mixing the two idler beams into a single mode, the same idler state is related to different photon distribution between the signal fields. These states are then superimposed, giving rise to coherence. This occurs despite that the intensity of the idler beam com-

\footnotetext{
*Permanent address: Departamento de Optica, Facultad de Ciencias Físicas, Universidad Complutense, Madrid, Spain.
}

ing from the first crystal is too weak to induce any stimulated down-conversion in the second one. So, the induced coherence is not due to induced emission. The role of the vacuum fluctuations in this process also has been discussed [8].

The purpose of this work is to study how this transformation of entanglement into coherence is reflected in the field state. Nonlinear processes are well known as producing nonclassical states of light with interesting properties [9]. Here we intend to study which states are generated in this arrangement. To this end, we mainly examine the state in the signal beams when a photon-number measurement has been carried out in the idler beam. Then, we can both analyze the properties of the field generated and show which kind of states can be produced. We will do this when the idler beams are completely or partially connected. We also extend this analysis to photon-number measurements in the signal modes.

\section{COMPLETE COUPLING OF THE IDLER MODES}

The situation to be studied is illustrated in Fig. 1, which shows the two nonlinear crystals, NL1 and NL2, whose idler modes we will assume are perfectly superimposed and aligned. A lossless beam splitter, BS, can be inserted in the idler path between the two crystals to control the degree of connection between the two idler modes.

In this section we will consider that the beam splitter is removed. We are then in the best conditions to observe the induced coherence between the signal modes. If we combine them by means of a beam splitter a second-order interference arises, because there would be no way of telling which crys-

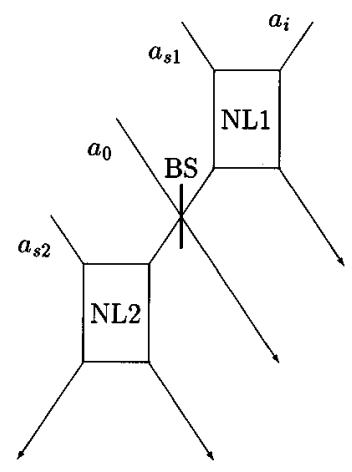

FIG. 1. Outline of the arrangement under discussion illustrating the definition of the relevant field amplitudes. 
tal emitted the photons recorded after the beam splitter. A photon-number measurement by means of a detector placed in the idler beam after the two crystals would not distinguish whether the emission has taken place in one or the other. Accordingly, the corresponding probability amplitudes of the possible processes are added, giving rise to the interference.

Our purpose here is the study of the characteristics and properties of the field in the signal modes.

The parametric interaction at the two nonlinear media is described by the effective interaction Hamiltonians $H_{1}$ and $\mathrm{H}_{2}$,

$$
H_{j}=\hbar\left(g_{j} a_{s j}^{\dagger} a_{i}^{\dagger}+g_{j}^{*} a_{s j} a_{i}\right)
$$

with $j=1,2$, where $a_{s j}$ and $a_{i}$ are the annihilation operators for the corresponding signal and idler beams, and $g_{j}$ is a parameter depending on the pump, which is assumed to be strong, classical, and coherent, and the nonlinear characteristics of the media. The relation between the input $|\psi\rangle$ and output $|\bar{\psi}\rangle$ field states of the whole device is provided by the unitary transformation $T$ (it will not be necessary to take into account explicitly the free propagation of the idler field between NL1 and NL2),

$$
T=T_{2} T_{1}=\exp \left(-i H_{2} \tau_{2} / \hbar\right) \exp \left(-i H_{1} \tau_{1} / \hbar\right)
$$

where $\tau_{j}$ are the corresponding interaction times, such that $|\bar{\psi}\rangle=T|\psi\rangle$.

We will start considering that the input field is vacuum $|\psi\rangle=|0\rangle_{s 1}|0\rangle_{s 2}|0\rangle_{i}$. The expression for the output state can be obtained (since the input state is vacuum) using the disentangling theorem for $T_{2}$ and $T_{1}$ [10], for instance,

$$
\begin{aligned}
T_{j}= & \frac{1}{\mu_{j}} \exp \left(\frac{\nu_{j}}{\mu_{j}} a_{s j}^{\dagger} a_{i}^{\dagger}\right) \exp \left[-\ln \mu_{j}\left(a_{s j}^{\dagger} a_{s j}+a_{i}^{\dagger} a_{i}\right)\right] \\
& \times \exp \left(-\frac{\nu_{j}^{*}}{\mu_{j}} a_{s j} a_{i}\right),
\end{aligned}
$$

where

$$
\mu_{j}=\cosh \left|g_{j}\right| \tau_{j}, \quad \nu_{j}=-i \frac{g_{j}}{\left|g_{j}\right|} \sinh \left|g_{j}\right| \tau_{j}
$$

The output field $|\bar{\psi}\rangle=T|\psi\rangle$ is in the number basis

$$
\begin{aligned}
|\bar{\psi}\rangle= & \sum_{m, k=0}^{\infty}\left(\begin{array}{c}
m+k \\
k
\end{array}\right)^{1 / 2} \frac{1}{\mu_{1} \mu_{2}^{m+1}}\left(\frac{\nu_{1}}{\mu_{1}}\right)^{m}\left(\frac{\nu_{2}}{\mu_{2}}\right)^{k} \\
& \times|m\rangle_{s 1}|k\rangle_{s 2}|m+k\rangle_{i} .
\end{aligned}
$$

We can note the symmetry present between the two signal modes after the alignment of the idler modes.

Now let us assume that a photon-number measurement is performed in the idler beam, $n$ being the outcome. The state in the signal mode after this measurement $\left|\bar{\psi}_{n}\right\rangle$ is given by the projection of the number state $|n\rangle_{i}$ on $|\bar{\psi}\rangle$, giving

$$
\left|\bar{\psi}_{n}\right\rangle={ }_{i}\langle n \mid \bar{\psi}\rangle=\frac{1}{\left(1+|\xi|^{2}\right)^{n / 2}} \sum_{m=0}^{n}\left(\begin{array}{c}
n \\
m
\end{array}\right)^{1 / 2} \xi^{m}|m\rangle_{s 1}|n-m\rangle_{s 2},
$$

where $\xi=\nu_{1} /\left(\mu_{1} \nu_{2}\right)$.

It can be seen that this is an $\mathrm{SU}(2)$ coherent state $[10,11]$. It is a coherent state corresponding to the realization of the algebra of this group in terms of the bosonic operators

$$
\begin{aligned}
& J_{x}=\frac{1}{2}\left(a_{s 1}^{\dagger} a_{s 2}+a_{s 1} a_{s 2}^{\dagger}\right), \\
& J_{y}=\frac{i}{2}\left(a_{s 1} a_{s 2}^{\dagger}-a_{s 1}^{\dagger} a_{s 2}\right), \\
& J_{z}=\frac{1}{2}\left(a_{s 1}^{\dagger} a_{s 1}-a_{s 2}^{\dagger} a_{s 2}\right),
\end{aligned}
$$

being the Casimir operator

$$
J^{2}=\frac{N}{2}\left(\frac{N}{2}+1\right)
$$

with $N=a_{s 1}^{\dagger} a_{s 1}+a_{s 2}^{\dagger} a_{s 2}$ the total photon number in the signal modes.

Then we have that the state on the signal modes $\left|\bar{\psi}_{n}\right\rangle$ can be expressed as

$$
\begin{aligned}
\left|\bar{\psi}_{n}\right\rangle & =\exp \left(\frac{\theta}{2} e^{-i \phi} a_{s 1}^{\dagger} a_{s 2}-\frac{\theta}{2} e^{i \phi} a_{s 1} a_{s 2}^{\dagger}\right)|0\rangle_{s 1}|n\rangle_{s 2} \\
& =\frac{1}{\left(1+|\xi|^{2}\right)^{n / 2}} \exp \left(\xi a_{s 1}^{\dagger} a_{s 2}\right)|0\rangle_{s 1}|n\rangle_{s 2},
\end{aligned}
$$

where $\xi=\tan (\theta / 2) e^{-i \phi}$.

It is worth comparing this state with the one that would be obtained if the idler modes were not superimposed. A similar photon-number measurement, now in the two idler modes, would reduce the signal modes to a product of number states and the second-order interference would be lost. The breaking of the connection would lead to the possibility of distinguishing the nonlinear crystal where the emission takes place, and the route followed by the photons incident at the beam splitter mixing the signal modes. This knowledge wipes out the interference effect.

The origin of this coherence as indistinguishability is somewhat reflected in the photon-number probabilities in the signal modes in (2.6), which turns out to be binomial, corresponding to the random distribution of $n$ indistinguishable particles between the two modes. It occurs in the same terms that it does in a beam splitter over an incident state of $n$ photons in one of the input ports and vacuum in the other.

After that we can express the complete output field before the measurement as a kind of superposition of SU(2) coherent states,

$$
|\bar{\psi}\rangle=\sum_{n=0}^{\infty} \frac{1}{\mu}\left(\frac{\nu}{\mu}\right)^{n}|n\rangle_{i}\left|\bar{\psi}_{n}\right\rangle
$$

where $\mu=\mu_{1} \mu_{2}$ and $\nu=\sqrt{\mu_{1}^{2} \mu_{2}^{2}-1} \nu_{2} /\left|\nu_{2}\right|$. It must be noted that all the $\mathrm{SU}(2)$ coherent states $\left|\bar{\psi}_{n}\right\rangle$ are characterized by the same parameter $\xi$ since it does not depend on $n$.

Before continuing let us use another way to identify the reduced state after the measurement, which can be useful 
when the state or its properties are not easily recognizable from its expression in the number basis. It can be used when the input states are eigenstates of a given set of operators. The purpose is to use the eigenvalue equations and, by removing the idler mode in terms of the measurement, to reduce the problem to a different set of eigenvalue equations for the signal modes only [5]. In this case the input state is an eigenstate of the three annihilation operators. Using this fact we have that the output state must verify the equations

$$
\begin{gathered}
T a_{s 1} T^{\dagger}|\bar{\psi}\rangle=\left(\mu_{1} a_{s 1}+\nu_{1} \nu_{2}^{*} a_{s 2}-\nu_{1} \mu_{2} a_{i}^{\dagger}\right)|\bar{\psi}\rangle=0, \\
T a_{s 2} T^{\dagger}|\bar{\psi}\rangle=\left(\mu_{2} a_{s 2}-\nu_{2} a_{i}^{\dagger}\right)|\bar{\psi}\rangle=0, \\
T a_{i} T^{\dagger}|\bar{\psi}\rangle=\left(\mu_{1} \mu_{2} a_{i}-\nu_{1} a_{s 1}^{\dagger}-\mu_{1} \nu_{2} a_{s 2}^{\dagger}\right)|\bar{\psi}\rangle=0 .
\end{gathered}
$$

Since we are interested here in a measurement in the idler mode we can isolate the action of the idler operators

$$
\begin{gathered}
a_{i}^{\dagger}|\bar{\psi}\rangle=\frac{1}{\nu_{1} \mu_{2}}\left(\mu_{1} a_{s 1}+\nu_{1} \nu_{2}^{*} a_{s 2}\right)|\bar{\psi}\rangle=\frac{\mu_{2}}{\nu_{2}} a_{s 2}|\bar{\psi}\rangle, \\
a_{i}|\bar{\psi}\rangle=\frac{1}{\mu_{1} \mu_{2}}\left(\nu_{1} a_{s 1}^{\dagger}+\mu_{1} \nu_{2} a_{s 2}^{\dagger}\right)|\bar{\psi}\rangle .
\end{gathered}
$$

We can note from the first equation that the relation

$$
\left(a_{s 1}-\frac{\nu_{1}}{\mu_{1} \nu_{2}} a_{s 2}\right)|\bar{\psi}\rangle=0
$$

is verified irrespective of any other condition. With (2.12) we could obtain the reduced eigenvalue equations for the signal states after any measurement in the idler mode, simply by constructing the corresponding measured operator and projecting over its eigenvectors. Since we are interested in the measurement of the photon number we can write

$$
\begin{aligned}
a_{i}^{\dagger} a_{i}|\bar{\psi}\rangle= & \frac{1}{\mu_{1} \nu_{2}}\left(\nu_{1} a_{s 1}^{\dagger}+\mu_{1} \nu_{2} a_{s 2}^{\dagger}\right) a_{s 2}|\bar{\psi}\rangle \\
= & \frac{1}{\nu_{1} \mu_{2}^{2} \mu_{1}}\left(\nu_{1} a_{s 1}^{\dagger}+\mu_{1} \nu_{2} a_{s 2}^{\dagger}\right) \\
& \times\left(\mu_{1} a_{s 1}+\nu_{1} \nu_{2}^{*} a_{s 2}\right)|\bar{\psi}\rangle .
\end{aligned}
$$

Finally, we can project these two equations over a photonnumber state in the idler mode. With the help of (2.13) we can express the result in different ways. We have, for instance,

$$
\begin{gathered}
\left(a_{s 1}^{\dagger} a_{s 1}+a_{s 2}^{\dagger} a_{s 2}\right)\left|\bar{\psi}_{n}\right\rangle=n\left|\bar{\psi}_{n}\right\rangle \\
\left(a_{s 2}^{\dagger} a_{s 2}-a_{s 1}^{\dagger} a_{s 1}+2 \frac{\nu_{1}}{\mu_{1} \nu_{2}} a_{s 1}^{\dagger} a_{s 2}\right)\left|\bar{\psi}_{n}\right\rangle=n\left|\bar{\psi}_{n}\right\rangle
\end{gathered}
$$

or also

$$
\begin{gathered}
\left(a_{s 1}^{\dagger} a_{s 1}+a_{s 2}^{\dagger} a_{s 2}\right)\left|\bar{\psi}_{n}\right\rangle=n\left|\bar{\psi}_{n}\right\rangle \\
\left(\frac{\mu_{1} \nu_{2}}{\nu_{1}} a_{s 2}^{\dagger} a_{s 1}+\frac{\nu_{1}}{\mu_{1} \nu_{2}} a_{s 1}^{\dagger} a_{s 2}\right)\left|\bar{\psi}_{n}\right\rangle=n\left|\bar{\psi}_{n}\right\rangle,
\end{gathered}
$$

that define an SU(2) coherent state. The first equations in (2.15) and (2.16) express that, since the idler and signal photons are produced in pairs, there are as many signal photons as idler photons have been detected. The other one characterizes the state as coherent $\mathrm{SU}(2)$ within the subspace of total signal photon number $n$. This can be seen noting that (2.13) and the first equations in (2.15) and (2.16) can be written as

$$
\begin{gathered}
U\left(a_{s 1}^{\dagger} a_{s 1}+a_{s 2}^{\dagger} a_{s 2}\right) U^{\dagger}\left|\bar{\psi}_{n}\right\rangle=n\left|\bar{\psi}_{n}\right\rangle, \\
U a_{s 1} U^{\dagger}\left|\bar{\psi}_{n}\right\rangle=0,
\end{gathered}
$$

where $U$ is an $\mathrm{SU}(2)$ unitary transformation, specifically that in (2.9), and then we have $U^{\dagger}\left|\bar{\psi}_{n}\right\rangle=|0\rangle_{s 1}|n\rangle_{s 2}$. The second equation characterizes the output state as a superposition of SU(2) coherent states with the same parameter $\xi$ and different values of the Casimir operator $\mathrm{J}^{2}$. The first expression fixes in this case its value to $(n / 2)(n / 2+1)$.

As we have mentioned, this procedure leading to (2.15) or (2.16) is especially useful when the expression for the output state is involved or not easily recognizable. We can find it useful, for instance, when the input state is coherent, $|\psi\rangle=\left|\alpha_{s 1}\right\rangle_{s 1}\left|\alpha_{s 2}\right\rangle_{s 2}\left|\alpha_{i}\right\rangle_{i}$. We again assume that the photon number is measured in the output idler beam, giving the outcome $n$. Since the input state is also an eigenstate of the annihilation operators, the procedure followed from (2.11) to (2.14) can be also applied to this case by simply replacing 0 by $\alpha_{s 1}, \alpha_{s 2}$, and $\alpha_{i}$ in the right-hand side of (2.11). Following the same steps we arrive at equations defining the output signal state after the measurement $\left|\bar{\psi}_{n, \alpha}\right\rangle$, which can be written in the form

$$
\begin{gathered}
\left(a^{\prime \prime}{ }_{s 1}^{\dagger} a_{s 1}^{\prime}+a^{\prime \dagger}{ }_{s 2}^{\prime} a_{s 2}^{\prime}+\alpha_{i}^{\prime} a_{s 2}^{\prime}\right)\left|\bar{\psi}_{n, \alpha}\right\rangle=n\left|\bar{\psi}_{n, \alpha}\right\rangle \\
\left(\frac{\mu_{1} \nu_{2}}{\nu_{1}} a^{\prime \dagger}{ }_{s 2}^{\dagger} a_{s 1}^{\prime}+\frac{\nu_{1}}{\mu_{1} \nu_{2}} a_{s 1}^{\prime \dagger} a_{s 2}^{\prime}+\alpha_{i}^{\prime} a_{s 2}^{\prime}\right)\left|\bar{\psi}_{n, \alpha}\right\rangle=n\left|\bar{\psi}_{n, \alpha}\right\rangle
\end{gathered}
$$

where $a_{s 1}^{\prime}=a_{s 1}-\alpha_{s 1}^{\prime}$ and $a_{s 2}^{\prime}=a_{s 2}-\alpha_{s 2}^{\prime}$ are displaced annihilation operators and

$$
\begin{gathered}
\alpha_{s 1}^{\prime}=\frac{1}{\mu_{1}}\left(\alpha_{s 1}-\frac{\nu_{1} \nu_{2}^{*}}{\mu_{2}} \alpha_{s 2}\right), \\
\alpha_{s 2}^{\prime}=\frac{\alpha_{s 2}}{\mu_{2}}, \\
\alpha_{i}^{\prime}=\frac{1}{\mu_{1} \nu_{2}}\left(\alpha_{i}+\frac{\nu_{1}}{\mu_{1}} \alpha_{s 1}^{*}+\frac{\nu_{2}}{\mu_{1} \mu_{2}} \alpha_{s 2}^{*}\right) .
\end{gathered}
$$

It can be seen from (2.18) that there is a close relation between this state and the corresponding one when the input state was vacuum, which is a particular example of this case with $\alpha=\alpha^{\prime}=0$. Since

$$
\exp \left(\alpha a_{s 2}\right) a_{s 2}^{\dagger} \exp \left(-\alpha a_{s 2}\right)=a_{s 2}^{\dagger}+\alpha
$$

their relation can be established by means of the composition of a unitary and a nonunitary transformation in the form 


$$
\left|\bar{\psi}_{n, \alpha}\right\rangle=\mathscr{N} D_{s 1}\left(\alpha_{s 1}^{\prime}\right) D_{s 2}\left(\alpha_{s 2}^{\prime}\right) \exp \left(\alpha_{i}^{\prime} a_{s 2}\right)\left|\bar{\psi}_{n, \alpha=0}\right\rangle
$$

where $D_{j}\left(\alpha^{\prime}\right)=\exp \left(\alpha^{\prime} a_{j}^{\dagger}-\alpha^{\prime *} a_{j}\right)$ is the unitary displacement operator acting in the $j$ mode and $\mathscr{N}$ is a normalization constant.

Going back to the case with the input vacuum state we can ask whether a similar result holds if the measurement is performed in one of the signal modes instead of the idler mode. Then, let us study which is the state that arises in the $a_{s 1}$ and $a_{i}$ modes when we interrupt the second signal beam with a photodetector. If the result of such a measurement is $n$ the reduced state in the other two modes is

$$
\begin{aligned}
\left|\bar{\psi}_{n}\right\rangle & ={ }_{s 2}\langle n \mid \bar{\psi}\rangle \\
& =\left(1-|\eta|^{2}\right)^{(n+1) / 2} \sum_{m=0}^{\infty}\left(\begin{array}{c}
n+m \\
m
\end{array}\right)^{1 / 2} \eta^{m}|m\rangle_{s 1}|n+m\rangle_{i},
\end{aligned}
$$

where $\eta=\nu_{1} /\left(\mu_{1} \mu_{2}\right)$.

This state can be recognized as an $\mathrm{SU}(1,1)$ coherent state [10], corresponding to the realization of the algebra of this group in terms of the bosonic operators

$$
\begin{gathered}
K_{x}=\frac{1}{2}\left(a_{s 1}^{\dagger} a_{i}^{\dagger}+a_{s 1} a_{i}\right), \\
K_{y}=\frac{i}{2}\left(a_{s 1} a_{i}-a_{s 1}^{\dagger} a_{i}^{\dagger}\right), \\
K_{z}=\frac{1}{2}\left(a_{s 1}^{\dagger} a_{s 1}+a_{i}^{\dagger} a_{i}+1\right),
\end{gathered}
$$

being the Casimir operator

$$
K_{z}^{2}-K_{x}^{2}-K_{y}^{2}=\frac{K}{2}\left(\frac{K}{2}+1\right),
$$

with $K=a_{s 1}^{\dagger} a_{s 1}-a_{i}^{\dagger} a_{i}-1$.

Then we have that the state (2.22) can be expressed as

$$
\begin{aligned}
\left|\bar{\psi}_{n}\right\rangle & =\exp \left(\bar{g} a_{s 1}^{\dagger} a_{i}^{\dagger}-\bar{g}^{*} a_{s 1} a_{i}\right)|0\rangle_{s 1}|n\rangle_{i} \\
& =\left(1-|\eta|^{2}\right)^{(n+1) / 2} \exp \left(\eta a_{s 1}^{\dagger} a_{i}^{\dagger}\right)|0\rangle_{s 1}|n\rangle_{i},
\end{aligned}
$$

where $\eta=(\bar{g} /|\bar{g}|) \tanh |\bar{g}|$.

This leads to writing the whole output state (2.5) also as a superposition of $\mathrm{SU}(1,1)$ coherent states, all of them characterized by the same parameter $\eta$ involved in (2.25),

$$
|\bar{\psi}\rangle=\sum_{n=0}^{\infty} \frac{1}{\mu}\left(\frac{\nu}{\mu}\right)^{n}|n\rangle_{s 2}\left|\bar{\psi}_{n}\right\rangle
$$

where now $\nu=\nu_{2} \mu_{1}$ and $\mu=\sqrt{\mu_{1}^{2}\left|\nu_{2}\right|^{2}+1}$. It can be noted that there would be no qualitative difference in this case between interrupting the first or the second signal modes by means of a photon-number measurement. If such a measurement is carried out in the first signal mode, giving the outcome $n$, we would then have a number state $|n\rangle_{i}$ incident on the second crystal, giving rise to an $\mathrm{SU}(1,1)$ coherent state
$T_{2}|0\rangle_{s 2}|n\rangle_{i}$ in the output idler and second signal beams. This fact can be explained by the similar role played by the signal beams after the alignment of the idlers.

This result could be also derived by the same procedure followed from (2.11) to (2.14) but now particularized to a measurement in the second signal mode. In this procedure it appears that the equation

$$
\left(a_{s 1}-\frac{\nu_{1}}{\mu_{1} \mu_{2}} a_{i}^{\dagger}\right)|\bar{\psi}\rangle=0
$$

is satisfied irrespective of any measurement on the field. Here again, in view of (2.25), it can be seen that this condition characterizes the state as a superposition of $\mathrm{SU}(1,1)$ coherent states with the same parameter $\eta$ and different values of the Casimir operator.

With the help of this last relation, the equations defining the output field conditioned to the measurement of the photon number in the second signal beam can be written in different ways. We have

$$
\begin{gathered}
\left(a_{i}^{\dagger} a_{i}-a_{s 1}^{\dagger} a_{s 1}\right)\left|\bar{\psi}_{n}\right\rangle=n\left|\bar{\psi}_{n}\right\rangle, \\
\left(a_{s 1}^{\dagger} a_{s 1}+a_{i}^{\dagger} a_{i}-2 \frac{\nu_{1}}{\mu_{1} \mu_{2}} a_{s 1}^{\dagger} a_{i}^{\dagger}\right)\left|\bar{\psi}_{n}\right\rangle=n\left|\bar{\psi}_{n}\right\rangle,
\end{gathered}
$$

or also

$$
\begin{gathered}
\left(a_{i}^{\dagger} a_{i}-a_{s 1}^{\dagger} a_{s 1}\right)\left|\bar{\psi}_{n}\right\rangle=n\left|\bar{\psi}_{n}\right\rangle, \\
\left(\frac{\mu_{1} \mu_{2}}{\nu_{1}} a_{i} a_{s 1}-\frac{\nu_{1}}{\mu_{1} \mu_{2}} a_{s 1}^{\dagger} a_{i}^{\dagger}\right)\left|\bar{\psi}_{n}\right\rangle=(n+1)\left|\bar{\psi}_{n}\right\rangle,
\end{gathered}
$$

both defining the $\mathrm{SU}(1,1)$ coherent state $(2.25)$. This can be seen by noting that (2.27) and the first equations in (2.28) and (2.29) can be written as

$$
\begin{gathered}
U\left(a_{i}^{\dagger} a_{i}-a_{s 1}^{\dagger} a_{s 1}\right) U^{\dagger}\left|\bar{\psi}_{n}\right\rangle=n\left|\bar{\psi}_{n}\right\rangle, \\
U a_{s 1} U^{\dagger}\left|\bar{\psi}_{n}\right\rangle=0,
\end{gathered}
$$

where $U$ is an $\mathrm{SU}(1,1)$ unitary transformation, specifically that in (2.25), and then we have $U^{\dagger}\left|\bar{\psi}_{n}\right\rangle=|0\rangle_{s 1}|n\rangle_{i}$.

Finally we consider again the case when the input state is coherent $|\psi\rangle=\left|\alpha_{s 1}\right\rangle_{s 1}\left|\alpha_{s 2}\right\rangle_{s 2}\left|\alpha_{i}\right\rangle_{i}$. The same procedure leads to equations for the reduced state $\left|\bar{\psi}_{n, \alpha}\right\rangle$ that can be written as

$$
\begin{aligned}
& \left(a_{i}^{\prime \dagger} a_{i}^{\prime}-a^{\prime \dagger}{ }_{s 1}^{\dagger} a_{s 1}^{\prime}-\alpha_{s 2}^{\prime} a_{i}^{\prime \dagger}\right)\left|\bar{\psi}_{n, \alpha}\right\rangle=n\left|\bar{\psi}_{n, \alpha}\right\rangle, \\
& \left(\frac{\mu_{1} \mu_{2}}{\nu_{1}} a_{s 1}^{\prime} a_{i}^{\prime}-\frac{\nu_{1}}{\mu_{1} \mu_{2}} a_{s 1}^{\prime \dagger} a_{i}^{\prime \dagger}-\alpha_{s 2}^{\prime} a_{i}^{\prime \dagger}\right)\left|\bar{\psi}_{n, \alpha}\right\rangle \\
& =(n+1)\left|\bar{\psi}_{n, \alpha}\right\rangle,
\end{aligned}
$$

where $a_{s 1}^{\prime}=a_{s 1}-\alpha_{s 1}^{\prime}$ and $a_{i}^{\prime}=a_{i}-\alpha_{i}^{\prime}$ are displaced annihilation operators and

$$
\alpha_{s 1}^{\prime}=\frac{1}{\mu_{1}}\left(\alpha_{s 1}-\frac{\nu_{1} \mu_{2}}{\nu_{2}} \alpha_{s 2}\right),
$$




$$
\begin{gathered}
\alpha_{i}^{\prime}=-\frac{\alpha_{s 2}^{*}}{\nu_{2}^{*}} \\
\alpha_{s 2}^{\prime}=\frac{1}{\mu_{1} \mu_{2}}\left(\alpha_{i}+\frac{\nu_{1}}{\mu_{1}} \alpha_{s 1}^{*}+\frac{\mu_{2}}{\mu_{1} \nu_{2}^{*}} \alpha_{s 2}^{*}\right) .
\end{gathered}
$$

The solution of (2.31) can be written again as the composition of a unitary and a nonunitary transformation acting to the output state when the incident one is the vacuum in the form

$$
\left|\bar{\psi}_{n, \alpha}\right\rangle=\mathscr{N} D_{s 1}\left(\alpha_{s 1}^{\prime}\right) D_{i}\left(\alpha_{i}^{\prime}\right) \exp \left(\alpha_{s 2}^{\prime} a_{i}^{\dagger}\right)\left|\bar{\psi}_{n, \alpha=0}\right\rangle .
$$

\section{PARTIAL COUPLING OF THE IDLER MODES}

The second-order coherence between the signal modes originates because of the impossibility of knowing in which crystal the emission takes place. We have shown how this fact is reflected in the field state. Any modification of the arrangement considered in the previous section leading to the possibility of knowledge about how the emitted photons are distributed between the two signal modes will wipe out the second-order interference effect.

One way to do this is by inserting a beam splitter in the idler beam between the two crystals. This means the possibility of obtaining some information about whether the emission takes place in NL1 or NL2 by putting a detector at the output of the beam splitter.

It has been pointed out that it is not necessary that this measurement is actually made. It is sufficient that after the insertion of the beam splitter the two photon paths become distinguishable.

Our purpose here is essentially the same as that followed in the previous section. We intend to study the effect of the entanglement and the idler coupling in the state of the field. We again will do this by asking for the field state after a number measurement in some of the modes. In the previous section we showed that the complete connection of the idler modes gives rise to coherent states $[\mathrm{SU}(2)$ and $\mathrm{SU}(1,1)]$, contrary to the case of idler modes completely decoupled, which originates number states. In this case of partial coupling we can expect that states somewhat intermediate between number and coherent states will appear. Their study is the aim of this section.

We assume that a beam splitter has been placed as is shown in Fig. 1, coupling the output idler mode of the first crystal with another mode we will describe by the annihilation operator $a_{0}$. Moreover, we will consider that a photodetector can be placed at the output of this beam splitter. The beam splitter will be described by real reflection $r$ and transmission $t$ coefficients with a $\pi$ phase change in one of the reflections. Its action on the field state is given by the unitary operator

$$
T_{\mathrm{BS}}=\exp \left[\gamma\left(a_{0}^{\dagger} a_{i}-a_{i}^{\dagger} a_{0}\right)\right]
$$

where $\tan \gamma=r / t$

The relation between the states entering $|\psi\rangle$ and leaving $|\bar{\psi}\rangle$ the whole device is now given by the transformation $T=T_{2} T_{\mathrm{BS}} T_{1}$. We will always consider that the input state is vacuum in all the modes. The output state, using, for instance, the corresponding disentangling theorems, is given by

$$
\begin{aligned}
|\bar{\psi}\rangle= & \sum_{m, n=0}^{\infty} \sum_{k=0}^{n}\left(\begin{array}{c}
m+k \\
m
\end{array}\right)^{1 / 2}\left(\begin{array}{c}
n \\
k
\end{array}\right)^{1 / 2} \frac{1}{\mu_{1} \mu_{2}^{k+1}}\left(\frac{\nu_{1}}{\mu_{1}}\right)^{n}\left(\frac{\nu_{2}}{\mu_{2}}\right)^{m} \\
& \times t^{k} r^{n-k}|n\rangle_{s 1}|m\rangle_{s 2}|m+k\rangle_{i}|n-k\rangle_{0} .
\end{aligned}
$$

Let us start by studying first the case corresponding to a measurement of the photon number in the $a_{0}$ and $a_{i}$ modes by means of photodetectors placed at the output of the beam splitter and at the output idler beam. If the result of such a measurement is $n_{0}$ and $n_{i}$, respectively, the state in the signal modes $\left|\bar{\psi}_{n_{i}, n_{0}}\right\rangle$ is given by the projection of (3.2) on $\left|n_{i}\right\rangle_{i}\left|n_{0}\right\rangle_{0}$. Although it is not difficult to extract the relevant information about the field state after this projection, we think it is more convenient to express the state originated in terms of an eigenvalue equation as we did in the previous section. The procedure is the same as followed there but now the starting point, instead of (2.11), is

$$
\begin{gathered}
T a_{s 1} T^{\dagger}|\bar{\psi}\rangle=\left(\mu_{1} a_{s 1}+t \nu_{1} \nu_{2}^{*} a_{s 2}-t \nu_{1} \mu_{2} a_{i}^{\dagger}-r \nu_{1} a_{0}^{\dagger}\right)|\bar{\psi}\rangle=0, \\
T a_{s 2} T^{\dagger}|\bar{\psi}\rangle=\left(\mu_{2} a_{s 2}-\nu_{2} a_{i}^{\dagger}\right)|\bar{\psi}\rangle=0, \\
T a_{i} T^{\dagger}|\bar{\psi}\rangle=\left(t \mu_{1} \mu_{2} a_{i}+r \mu_{1} a_{0}-\nu_{1} a_{s 1}^{\dagger}-t \mu_{1} \nu_{2} a_{s 2}^{\dagger}\right)|\bar{\psi}\rangle=0, \\
T a_{0} T^{\dagger}|\bar{\psi}\rangle=\left(-r \mu_{2} a_{i}+t a_{0}+r \nu_{2} a_{s 2}^{\dagger}\right)|\bar{\psi}\rangle=0 .
\end{gathered}
$$

The procedure leads to the following equations verified by the reduced state in the signal modes:

$$
\begin{aligned}
& \left(a_{s 1}^{\dagger} a_{s 1}+a_{s 2}^{\dagger} a_{s 2}\right)\left|\bar{\psi}_{n_{i}, n_{0}}\right\rangle=\left(n_{i}+n_{0}\right)\left|\bar{\psi}_{n_{i}, n_{0}}\right\rangle, \\
& \left(a_{s 2}^{\dagger} a_{s 2}-a_{s 1}^{\dagger} a_{s 1}+2 \frac{t \nu_{1}}{\mu_{1} \nu_{2}} a_{s 1}^{\dagger} a_{s 2}\right)\left|\bar{\psi}_{n_{i}, n_{0}}\right\rangle \\
& =\left(n_{i}-n_{0}\right)\left|\bar{\psi}_{n_{i}, n_{0}}\right\rangle .
\end{aligned}
$$

These equations can be solved for $\left|\bar{\psi}_{n_{i}, n_{0}}\right\rangle$ by getting the associated recurrence relation or by using the SU(2) commutation relations. In terms of the angular momentum operators (2.7) and (2.8) we can write (3.4) in the form

$$
\begin{gathered}
N\left|\bar{\psi}_{n_{i}, n_{0}}\right\rangle=\left(n_{0}+n_{i}\right)\left|\bar{\psi}_{n_{i}, n_{0}}\right\rangle, \\
\left(J_{z}-\xi J_{+}\right)\left|\bar{\psi}_{n_{i}, n_{0}}\right\rangle=\frac{n_{0}-n_{i}}{2}\left|\bar{\psi}_{n_{i}, n_{0}}\right\rangle,
\end{gathered}
$$

where $\quad J_{+}=J_{x}+i J_{y}=a_{s 1}^{\dagger} a_{s 2}, \quad$ and $\quad \xi=|\xi| \exp \left(i \delta_{\xi}\right)$ $=t \nu_{1} /\left(\mu_{1} \nu_{2}\right)$. Taking into account the commutation relations

$$
\left[J_{z}, J_{+}\right]=J_{+}, \quad\left[N, J_{+}\right]=0,
$$

we have

$$
J_{z}-\xi J_{+}=\exp \left(\xi J_{+}\right) J_{z} \exp \left(-\xi J_{+}\right)
$$

and then 


$$
\begin{aligned}
& \exp \left(\xi J_{+}\right) N \exp \left(-\xi J_{+}\right)\left|\bar{\psi}_{n_{i}, n_{0}}\right\rangle=\left(n_{0}+n_{i}\right)\left|\bar{\psi}_{n_{i}, n_{0}}\right\rangle \\
& \exp \left(\xi J_{+}\right) J_{z} \exp \left(-\xi J_{+}\right)\left|\bar{\psi}_{n_{i}, n_{0}}\right\rangle=\frac{n_{0}-n_{i}}{2}\left|\bar{\psi}_{n_{i}, n_{0}}\right\rangle
\end{aligned}
$$

which give the solution as

$$
\begin{aligned}
\left|\bar{\psi}_{n_{i}, n_{0}}\right\rangle & =\mathscr{N} \exp \left(\xi J_{+}\right)\left|n_{0}\right\rangle_{s 1}\left|n_{i}\right\rangle_{s 2} \\
& =\mathscr{N} \exp \left(\frac{t \nu_{1}}{\mu_{1} \nu_{2}} a_{s 1}^{\dagger} a_{s 2}\right)\left|n_{0}\right\rangle_{s 1}\left|n_{i}\right\rangle_{s 2},
\end{aligned}
$$

where $\mathscr{N}$ is a normalization constant.

The meaning of the first equation in (3.4) is again clear since the signal and idler photons are produced in pairs. The second one is very similar to the corresponding one in (2.15), but now it only defines an $\mathrm{SU}(2)$ coherent state in two particular cases, namely, $n_{i}=0$ or $n_{0}=0$.

In the $n_{i}=0$ case the state in the signal modes given by (3.9) is the number state $\left|n_{0}\right\rangle_{s 1}|0\rangle_{s 2}$. The result of the measurement shows that no photons have been generated in the second medium which gives complete information concerning the photons' path.

The case $n_{0}=0$ also gives a coherent state as can be seen comparing (3.9) with (2.9) or (3.4) with (2.15). This case corresponds to an (accidental) erasure of the photon path information provided by the detector at the output of the beam splitter. If it turns up that no photons are registered by this detector once again there is no information available about where the $n_{i}$ photons have been emitted and we return to the situation of the previous section.

In the general case $n_{i} \neq 0, n_{0} \neq 0$, these expressions also show that in the limit $t \rightarrow 0$ the state tends to be a number state with the corresponding loss of second-order coherence. This can be related with the increasing degree of knowledge about the number of photons emitted by each crystal. When $t=0$ the two idler beams are completely decoupled, giving a number state in the signal modes and the interference vanishes. On the other hand, when $t=1$ no photons can be registered at the output of the beam splitter and we get then the same states obtained in the previous section.

Next we show that these states (3.9) that are no longer coherent are nevertheless closely related with another particular set of states that are the minimum uncertainty states of the SU(2) operators (2.7). From (3.5) it can be seen [12] that the state $\left|\psi_{\text {min }}\right\rangle$, obtained from $\left|\bar{\psi}_{n_{i}, n_{0}}\right\rangle$ by means of an $\mathrm{SU}(2)$ transformation (that is, the action of a beam splitter),

$$
\left|\psi_{\min }\right\rangle=\exp \left(-i \theta J_{x}\right) \exp \left(-i \delta J_{z}\right)\left|\bar{\psi}_{n_{i}, n_{0}}\right\rangle,
$$

verifies the eigenvalue equation

$$
\left(J_{x}+i \lambda J_{y}\right)\left|\psi_{\min }\right\rangle=i \frac{n_{i}-n_{0}}{2} \sqrt{\lambda^{2}-1}\left|\psi_{\min }\right\rangle
$$

where the parameters in these equations are defined as $\lambda=\sqrt{1+|\xi|^{2}} /|\xi|, \quad \delta=\delta_{\xi}+\pi / 2$, and $\lambda \cos \theta=1$. This equation shows that the states $\left|\bar{\psi}_{n_{i}, n_{0}}\right\rangle$ arising in the arrangement we are studying are closely related with the minimum uncer- tainty states of the $\mathrm{SU}(2)$ operators [13]. These states have found application in the context of interferometric measurement because the SU(2) operators are what is measured there. The minimum character of these states, and their squeezing controlled by the parameter $\lambda$, is relevant concerning the accuracy of interferometric measurements [12]. We can note that the parameters characterizing these states do not depend on the result of the measurement as far as $\lambda, \theta$, and $\delta$ depend only on $\xi$.

In what follows we briefly discuss the kinds of states that arise when the photon-number measurement is performed in other pairs of modes. We will try to show whether their properties are similar to those encountered before.

Now we assume that the two detectors have been placed at the output signal modes. When the outcomes of such photon-number measurement are $n_{1}$ and $n_{2}$ for the first and second signal modes, respectively, the state in the $a_{i}$ and $a_{0}$ modes is given by the solution of the equations

$$
\begin{gathered}
\left(a_{i}^{\dagger} a_{i}+a_{0}^{\dagger} a_{0}\right)\left|\bar{\psi}_{n_{1}, n_{2}}\right\rangle=\left(n_{1}+n_{2}\right)\left|\bar{\psi}_{n_{1}, n_{2}}\right\rangle, \\
\left(a_{0}^{\dagger} a_{0}-a_{i}^{\dagger} a_{i}+2 \frac{t}{\mu_{2} r} a_{i}^{\dagger} a_{0}\right)\left|\bar{\psi}_{n_{1}, n_{2}}\right\rangle=\left(n_{1}-n_{2}\right)\left|\bar{\psi}_{n_{1}, n_{2}}\right\rangle,
\end{gathered}
$$

which are formally the same as in (3.4), so we need not discuss their properties further.

If the measurement is performed in the modes $a_{s 2}$ and $a_{0}$, giving $n_{2}$ and $n_{0}$, respectively, we have that the state in the $a_{i}, a_{s 1}$ modes is defined by

$$
\begin{aligned}
\left(a_{i}^{\dagger} a_{i}-a_{s 1}^{\dagger} a_{s 1}\right)\left|\bar{\psi}_{n_{2}, n_{0}}\right\rangle & =\left(n_{2}-n_{0}\right)\left|\bar{\psi}_{n_{2}, n_{0}}\right\rangle, \\
\left(a_{i}^{\dagger} a_{i}+a_{s 1}^{\dagger} a_{s 1}-2 \frac{t \nu_{1}}{\mu_{1} \mu_{2}} a_{s 1}^{\dagger} a_{i}^{\dagger}\right)\left|\bar{\psi}_{n_{2}, n_{0}}\right\rangle & \\
& =\left(n_{0}+n_{2}\right)\left|\bar{\psi}_{n_{2}, n_{0}}\right\rangle .
\end{aligned}
$$

Here again these equations can be solved by means of a recurrence relation or by using the $\mathrm{SU}(1,1)$ commutation relations. In terms of the corresponding operators (2.23) and (2.24) we can write (3.13) in the form

$$
\begin{gathered}
K\left|\bar{\psi}_{n_{2}, n_{0}}\right\rangle=\left(n_{0}-n_{2}-1\right)\left|\bar{\psi}_{n_{2}, n_{0}}\right\rangle, \\
\left(K_{z}-\eta K_{+}\right)\left|\bar{\psi}_{n_{2}, n_{0}}\right\rangle=\frac{n_{0}+n_{2}+1}{2}\left|\bar{\psi}_{n_{2}, n_{0}}\right\rangle,
\end{gathered}
$$

where $\quad K_{+}=K_{x}+i K_{y}=a_{s 1}^{\dagger} a_{i}^{\dagger}, \quad$ and $\quad \eta=|\eta| \exp \left(i \delta_{\eta}\right)$ $=t \nu_{1} /\left(\mu_{1} \mu_{2}\right)$. From the commutation relations

$$
\left[K_{z}, K_{+}\right]=K_{+}, \quad\left[K, K_{+}\right]=0,
$$

we have

$$
K_{z}-\eta K_{+}=\exp \left(\eta K_{+}\right) K_{z} \exp \left(-\eta K_{+}\right)
$$

and then 


$$
\begin{aligned}
& \exp \left(\eta K_{+}\right) K \exp \left(-\eta K_{+}\right)\left|\bar{\psi}_{n_{2}, n_{0}}\right\rangle=\left(n_{0}-n_{2}-1\right)\left|\bar{\psi}_{n_{2}, n_{0}}\right\rangle \\
& \exp \left(\eta K_{+}\right) K_{z} \exp \left(-\eta K_{+}\right)\left|\bar{\psi}_{n_{2}, n_{0}}\right\rangle=\frac{n_{0}+n_{2}+1}{2}\left|\bar{\psi}_{n_{2}, n_{0}}\right\rangle
\end{aligned}
$$

giving the solution

$$
\begin{aligned}
\left|\bar{\psi}_{n_{2}, n_{0}}\right\rangle & =\mathscr{N} \exp \left(\eta K_{+}\right)\left|n_{0}\right\rangle_{s 1}\left|n_{2}\right\rangle_{i} \\
& =\mathscr{N} \exp \left(\frac{t \nu_{1}}{\mu_{1} \mu_{2}} a_{s 1}^{\dagger} a_{i}^{\dagger}\right)\left|n_{0}\right\rangle_{s 1}\left|n_{2}\right\rangle_{i} .
\end{aligned}
$$

It can be seen by comparing (3.13) with (2.28) or (3.18) with (2.25) that these states are defined similarly to the SU(1,1) coherent states. They coincide when $n_{0}=0$ or $n_{2}=0$. The reason for this is similar to that discussed earlier.

We can ask whether these states, when $n_{0} \neq 0$ and $n_{2}$ $\neq 0$, can be related with minimum uncertainty states for the $\mathrm{SU}(1,1)$ operators $(2.24)$. We can define the state $\left|\psi_{\min }\right\rangle$, related to $\left|\bar{\psi}_{n_{2}, n_{0}}\right\rangle$ by means of an SU(1,1) transformation

$$
\left|\psi_{\min }\right\rangle=\exp \left(-i \theta K_{y}\right) \exp \left(-i \delta K_{z}\right)\left|\bar{\psi}_{n_{2}, n_{0}}\right\rangle,
$$

which verifies the eigenvalue equation [14]

$\left(K_{y}+i \lambda K_{z}\right)\left|\psi_{\min }\right\rangle=i \frac{n_{2}+n_{0}+1}{2} \sqrt{1+\lambda^{2}}\left|\psi_{\min }\right\rangle$,

where the parameters in these equations are defined as $\lambda=\sqrt{1-|\eta|^{2}} /|\eta|, \quad \delta=\delta_{\eta}$, and $\lambda \sinh \theta=1$.

Then, these equations show that the states $\left|\bar{\psi}_{n_{2}, n_{0}}\right\rangle$ in the $a_{i}, a_{s 1}$ modes in this case are related by means of an $\mathrm{SU}(1,1)$ transformation with minimum uncertainty states for the $K_{y}, K_{z}$ operators.

Finally, when we measure the photon number in the first signal and idler modes, with outcomes $n_{1}$ and $n_{i}$, respectively, the state in the $a_{s 2}$ and $a_{0}$ modes is given by

$$
\begin{gathered}
\left(a_{0}^{\dagger} a_{0}-a_{s 2}^{\dagger} a_{s 2}\right)\left|\bar{\psi}_{n_{1}, n_{i}}\right\rangle=\left(n_{1}-n_{i}\right)\left|\bar{\psi}_{n_{1}, n_{i}}\right\rangle, \\
\left(a_{0}^{\dagger} a_{0}+a_{s 2}^{\dagger} a_{s 2}+2 \frac{t}{r \nu_{2}} a_{0} a_{s 2}\right)\left|\bar{\psi}_{n_{1}, n_{i}}\right\rangle=\left(n_{1}+n_{i}\right)\left|\bar{\psi}_{n_{1}, n_{i}}\right\rangle .
\end{gathered}
$$

Following the same previous procedure in terms of the corresponding $\mathrm{SU}(1,1)$ operators for these modes we can write the equations for $\left|\bar{\psi}_{n_{1}, n_{i}}\right\rangle$ in the form

$$
\begin{gathered}
K\left|\bar{\psi}_{n_{1}, n_{i}}\right\rangle=\left(n_{1}-n_{i}-1\right)\left|\bar{\psi}_{n_{1}, n_{i}}\right\rangle, \\
\left(K_{z}+\eta K_{-}\right)\left|\bar{\psi}_{n_{1}, n_{i}}\right\rangle=\frac{n_{1}+n_{i}+1}{2}\left|\bar{\psi}_{n_{1}, n_{i}}\right\rangle,
\end{gathered}
$$

where $K_{-}=K_{x}-i K_{y}=a_{s 2} a_{0}$ and $\eta=t /\left(r \nu_{2}\right)$, giving the solution

$$
\begin{aligned}
\left|\bar{\psi}_{n_{1}, n_{i}}\right\rangle & =\mathscr{N} \exp \left(\eta K_{-}\right)\left|n_{i}\right\rangle_{s 2}\left|n_{1}\right\rangle_{0} \\
& =\mathscr{N} \exp \left(\frac{t}{r \nu_{2}} a_{s 2} a_{0}\right)\left|n_{i}\right\rangle_{s 2}\left|n_{1}\right\rangle_{0} .
\end{aligned}
$$

Here again we can ask whether there is a relation between these states (now spanned by a finite number of photonnumber states) and some special $\mathrm{SU}(1,1)$ states. Two cases should be distinguished: namely, $|\eta|>1$ and $|\eta|<1$. When $|\eta|<1$ we can define the state

$$
\left|\psi_{\min }\right\rangle=\exp \left(-i \theta K_{y}\right) \exp \left(i \delta K_{z}\right)\left|\bar{\psi}_{n_{1}, n_{i}}\right\rangle,
$$

which verifies the eigenvalue equation

$\left(K_{y}+i \lambda K_{z}\right)\left|\psi_{\text {min }}\right\rangle=i \frac{n_{1}+n_{i}+1}{2} \sqrt{1+\lambda^{2}}\left|\psi_{\text {min }}\right\rangle$,

where $\lambda=\sqrt{1-|\eta|^{2}} /|\eta|, \delta=\delta_{\eta}$, and $\lambda \sinh \theta=-1$. Then, after an $\mathrm{SU}(1,1)$ transformation we get minimum uncertainty for the $K_{y}, K_{z}$ operators. This is the same situation we found before and we arrive at the same state $\left|\psi_{\min }\right\rangle$.

The other case $|\eta|>1$ is different. In this case we can define in the same terms

$$
\left|\psi_{\min }\right\rangle=\exp \left(-i \theta K_{x}\right) \exp \left(i \delta K_{z}\right)\left|\bar{\psi}_{n_{1}, n_{i}}\right\rangle
$$

which verifies the eigenvalue equation

$\left(K_{x}-i \lambda K_{y}\right)\left|\psi_{\min }\right\rangle=-i \frac{n_{1}+n_{i}+1}{2} \sqrt{1-\lambda^{2}}\left|\psi_{\min }\right\rangle$,

where now we have $\lambda=\sqrt{|\eta|^{2}-1} /|\eta|, \quad \delta=\delta_{\eta}-\pi / 2$, and $\lambda \cosh \theta=1$. In this case we get $\mathrm{SU}(1,1)$ minimum uncertainty states for the $K_{x}, K_{y}$ operators, whose properties have been studied recently [14].

Finally we want to mention that it is not difficult to extend this analysis to the case when the input states are arbitrary coherent states instead of vacuum. In such a case we can get similar results to those in Sec. II. The states, arising from a number measurement, verify eigenvalue equations that are linear and quadratic in the annihilation and creation operators and therefore would be related with those studied here by means of unitary and nonunitary displacement operators.

\section{CONCLUSIONS}

We have analyzed the field states generated from the vacuum in two parametric down-converters whose idler beams are aligned. To this end we have derived eigenvalue equations for the field state conditioned to the result of a photon-number measurement in some of the modes. The conversion of entanglement into coherence in this arrangement is reflected in the appearance of SU(2) coherent states in the signal modes. These states appear in addition to the $\mathrm{SU}(1,1)$ coherent states associated with these nonlinear processes.

We have also studied how these states are modified after 
the insertion of a beam splitter in the idler path controlling the degree of connection of the two crystals. We have found that the states conditioned to a photon-number measurement are closely related with $\mathrm{SU}(2)$ and $\mathrm{SU}(1,1)$ minimum uncertainty states. Therefore, it seems that this process can provide a source for the generation of this kind of states.

\section{ACKNOWLEDGMENTS}

We would like to thank Professor V. Peřinová and Dr. A. Lukš for carefully reading the manuscript. A.L. also acknowledges the support of a grant from the Ministerio Español de Educación y Ciencia and the kind hospitality from the Optics Department of the Palacký University.
[1] J. Peřina, Z. Hradil, and B. Jurčo, Quantum Optics and Fundamentals of Physics (Kluwer, Dordrecht, 1994).

[2] P. H. S. Ribeiro, S. Pádua, J. C. Machado da Silva, and G. A. Barbosa, Phys. Rev. A 51, 1631 (1995).

[3] Y. Yamamoto, S. Machida, M. Imoto, M. Kitagawa, and G. Björk, J. Opt. Soc. Am. B 10, 1645 (1987).

[4] G. S. Agarwal, Quantum Opt. 2, 1 (1990).

[5] A. Lukš, V. Peřinová, and J. Křepelka, J. Mod. Opt. 41, 2325 (1994).

[6] X. Y. Zou, L. J. Wang, and L. Mandel, Phys. Rev. Lett. 67, 318 (1991); L. J. Wang, X. Y. Zou, and L. Mandel, Phys. Rev. A 44, 4614 (1991); X. Y. Zou, T. Grayson, G. A. Barbosa, and L. Mandel, ibid. 47, 2293 (1993).
[7] P. G. Kwiat, A. M. Steinberg, and R. Y. Chiao, Phys. Rev. A 49, 61 (1994).

[8] M. O. Scully and U. W. Rathe, Opt. Commun. 110, 373 (1994).

[9] J. Perina, Quantum Statisics of Linear and Nonlinear Optical Phenomena, 2nd ed. (Kluwer, Dordrecht, 1991).

[10] A. Perelomov, Generalized Coherent States and their Applications (Springer, Berlin, 1986).

[11] F. T. Arecchi, E. Courtens, R. Gilmore, and H. Thomas, Phys. Rev. A 6, 2211 (1972).

[12] M. Hillery and L. Mlodinow, Phys. Rev. A 48, 1548 (1993).

[13] C. Aragone, E. Chalbaud, and S. Salomo, J. Math. Phys. 17, 1963 (1976). K. Wódkiewicz and J. H. Eberly, J. Opt. Soc. Am. B 2, 458 (1985).

[14] C. C. Gerry and R. Grobe, Phys. Rev. A 51, 4123 (1995). 